\title{
Influenza Surveillance in the Department of Veterans Affairs (VA): 2012-2013 Influenza Season
}

\author{
Cynthia A. Lucero-Obusan*1,2, Mark Winters ${ }^{3,4}$, Patricia Schirmer ${ }^{1,2}$, Gina Oda ${ }^{1,2}$, Richard \\ A. Martinello ${ }^{1,5}$, Victoria J. Davey ${ }^{1}$ and Mark Holodniy ${ }^{1,2}$ \\ 1'Department of Veterans Affairs - Office of Public Health, Washington, DC, USA; ${ }^{2}$ VHA Office of Public Health Surveillance and \\ Research, Palo Alto, CA, USA; ${ }^{3}$ Stanford University, Stanford, CA, USA; ${ }^{V}$ VA Palo Alto Health Care System, Palo Alto, CA, USA; ${ }^{5}$ Yale \\ School of Medicine, New Haven, CT, USA
}

\section{Introduction}

Influenza is associated with significant morbidity and mortality nationally each year and VA's large elderly population is at particular risk. VA Office of Public Health (OPH) has monitored influenza and influenza-like-illness (ILI) activity using the VA's biosurveillance system since $2009(1,2)$. VA influenza surveillance capacity has expanded significantly in recent years to include inpatient influenza data, telephone triage data, laboratory testing data and enhanced geospatial mapping capabilities. Herein we summarize our ongoing influenza surveillance activities and describe the 2012-2013 influenza season activity in VA.

\section{Methods}

Influenza hospitalizations; ILI and influenza ICD-9 coded outpatient visits; total influenza testing and positive results; and influenza telephone triage calls for the 2012-2013 influenza season (9/30/125/18/13) were obtained using the VA Healthcare Associated Infection and Influenza Surveillance System (HAIISS) and compared to the 2 previous influenza seasons. Influenza vaccine procedure codes were captured from 9/1/12-5/18/13 since the 2012-2013 seasonal influenza vaccine was available in some locations prior to the 9/30/12 influenza season start date. A convenience sample of influenza positive nasopharyngeal specimens underwent hemagglutinin (HA) and neuraminidase (NA) gene sequencing. HA sequences were compared against the 2012-2013 vaccine strains.

\section{Results}

Over 200,000 Veterans were seen for ILI, with weekly percentages ranging from $1.6-5 \%$ (2.4\% overall for the season). Additional surveillance measures (Table 1) showed increases in outpatient visits, hospitalizations, telephone triage calls, total testing and positive influenza tests compared to previous seasons. The highest number of laboratory-confirmed influenza positive tests were seen in Texas (534), Mississippi (395), Tennessee (358), Florida (323) and California (279) (Figure 1).Over 1.89 million vaccinations were recorded, representing 29\% of 6.33 million patients treated in Fiscal Year 2012. For 2012-2013 season, VA OPH Reference Laboratory received 335 samples for analysis, of which 294 were positive for influenza. Of the H3N2, H1N1, and B specimens which were further characterized, amino acid changes were found in 2-5 of the HA gene epitopes among all strains. In total, 261 strains were tested for NA inhibitor resistance and none had drug resistance mutations (i.e. H275Y).

\section{Conclusions}

The 2012-2013 influenza season required more healthcare resource utilization and more Veterans $\geq 65$ years of age sought care compared to the last 2 seasons. Strain characterization demonstrated HA epitope differences compared to vaccine strains. Vaccination in VA could be improved upon.
Table 1. Selected VA Influenza Surveillance Metrics

\begin{tabular}{|c|c|c|c|}
\hline & $2010-2011$ & $2011-2012$ & $2012-2013$ \\
\hline Influenza-coded visits & 1,064 & 772 & 2,401 \\
\hline Influenza-coded hospitalizations & 767 & 373 & 2,219 \\
Patients aged $\geq 65$ & $369(48 \%)$ & $192(51 \%)$ & $1,398(63 \%)$ \\
\hline Influenza-coded telephone calls & 3,773 & 4,907 & 7,494 \\
\hline Influenza tests performed & 13,027 & 9,953 & 29,151 \\
Influenza positive & $1,632(13 \%)$ & $701(7 \%)$ & $4,921(17 \%)$ \\
Influenza A & $1,313(80 \%)$ & $644(92 \%)$ & $3,698(75 \%)$ \\
Influenza B & $304(19 \%)$ & $52(7 \%)$ & $1,180(24 \%)$ \\
Both A \& B or type not specified & $15(1 \%)$ & $5(1 \%)$ & $43(1 \%)$ \\
\hline
\end{tabular}

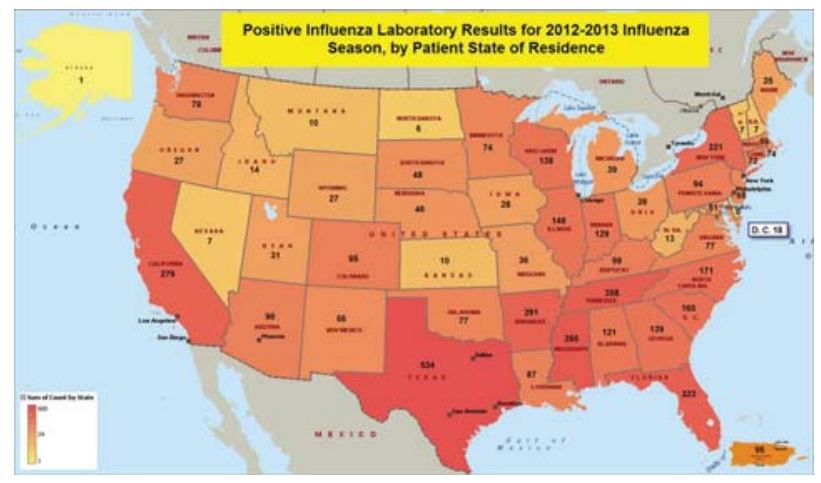

Figure 1. VA Laboratory-confirmed Positive Influenza Results, 2012-2013 Influenza Season

\section{Keywords}

Influenza; Surveillance; Veterans

\section{References}

1. Schirmer P, Lucero C, Oda G, Lopez J, Holodniy M. Effective detection of the 2009 H1N1 influenza pandemic in U.S. Veterans Affairs medical centers using a national electronic biosurveillance system. PLoS One 2010; 5(3):e9533.

2. Lucero C, Oda G, Cox K, Maldonado F, Lombardo J, Wojcik R, Holodniy M. Enhanced Health Event Detection and Influenza Surveillance Using a Joint Veterans Affairs and Department of Defense Biosurveillance Application. BMC Med Inform Decis Mak 2011; 11:56.

\section{*Cynthia A. Lucero-Obusan}

E-mail: cynthia.lucero@va.gov 